Verwundungen den Tod begïnstigt hatten, ist Schuld, dass die gehoffte Erledigung mancher wichtigen Streitfragen nunmehr eine neue ähnliche Gelegenheit abwarten muss.

Es ist mir eine angenehme Pflicht, dem Herrn Studiosus G. Kossiakowski an diesem Ort meinen besten Dank auszusprechen, für die liebenswürdige Beihülfe, welche er mir bei meinen gegenwärtigen Untersuchungen geleistet hat.

(Aus dem physiologischen Institute zu Christiania in Norwegen.)

\title{
Ueber angebliche Verbindungen des Traubenzuckers mit Kupferoxydhydrat.
}

Von

Prof. Worm Müller und Assistent J, Hagen.

Der Traubenzucker kann mit den Alkalien, Erdalkalien und, wie es scheint, mit den Oxyden einzelner schwerer Metalle, nämlich Blei und Kupfer, Verbindungen eingehen. Vom grössten Interesse wird es vielleicht aus naheliegenden Griunden sein, das Verhalten desselben zu Kupferoxyd resp. Kupferoxydhydrat zu studiren, und damit werden wir uns im Folgenden beschäftigen.

§. 1. Ueber die von Salkowski beschriebene Verbindung von Traubenzucker mit Kupferoxydhydrat.

Salkowski ${ }^{1}$ ) hat angegeben, dass es ihm gelungen sei, einen in Wasser unlöstichen blaugrünen Körper darzustellen, der auf 1. Mol. Zucker 5 Mol. Kupferoxydhydrat enthalte. Um diese Verbindung zu erlangen, setzt er zu $1 \mathrm{Mol}$. Zucker in Lösung $5 \mathrm{Mol}$. Kupfersulphat und $10 \mathrm{Mol}$. Natronhydrat; die ganze Zuckermenge werde dann in Verbindung mit allem Kupferoxyd ausgefällt, so

1) Dieses Archiv Bd. 6, 1872. S. 220 . 
Ueber angebl. Verbindungen d. Traubenzuckers mit Kupferoxydhydrat. 569

dass das neutrale Filtrat nur Natriumsulphat enthalte. Der Vorgang würde durch folgendes Schema auszudriicken sein:

$\mathrm{C}_{6} \mathrm{H}_{12} \mathrm{O}_{6}+5 \mathrm{CuSO}_{4}+10 \mathrm{NaOH}=\left[\mathrm{C}_{6} \mathrm{H}_{12} \mathrm{O}_{6}+5 \mathrm{Cu}(\mathrm{OH})_{2}\right]+5 \mathrm{Na}_{2} \mathrm{So}_{4}$.

Um die Richtigkeit dieser Angabe weiter darzuthun, fugte er einerseits eine verbältnissmässig grössere Menge Zucker hinzu; der Ueberschuss liess sich dann im Filtrate nachweisen. Gebranchte er andererseits eine grössere Menge des Kupfersulphats (und die damit aequivalente Quantität Natronhydrat), war der Niederschlag mit Oxydhydrat gemischt. Den Niederschlag selbst hat er seiner geringen Haltbarkeit wegen nicht direct analysirt.

Diese Verbindung nimmt in besonderem Grad das Interesse in Anspruch, schon desshalb, weil die quantitative Bestimmung des Traubenzuckers mittelst alkalischer Kupferoxydlösung darauf beruht, dass 1 Mol. Zucker 5 Mol. Kupferoxyd zu Oxydul reducirt. Es war daher wichtig, uns von der Richtigkeit jener Angabe zu überzeugen. Zu dem Ende haben wir die Versuche Salkowski's wiederholt, und sind dabei in schätzbarer Weise von Herrn Polytechniker Tobiesen unterstïtzt worden, der mit seiner reichen Erfahrung als Analytiker ein besonderes Interesse für physiologisehchemische Untersuchungen vereinigt.

Der bei den Versuchen angewendete Traubenzucker war im Institute nach der Methode von Schwarz dargestellt; er schmolz genau bei $146^{\circ}$, und seine Reinheit war ausserdem sowohl durch Elementaranalyse als auch mittelst des Circumpolarisationsapparates controllirt. Bei jedem Versuche wurden 0.9 gr Zucker, $6.235 \mathrm{gr}$ Kupfervitriol und $2.8065 \mathrm{gr} \mathrm{KOH} \mathrm{(50} \mathrm{cem} \mathrm{normaler} \mathrm{Kalilauge)} \mathrm{oder}$ $2.002 \mathrm{gr} \mathrm{NaOH}$ (25.66 cem einer 8.7\% igen NaOH-lösung) angewendet. Die Lösungen dieser Körper, welche immer mittelst frisch ausgekochten, destillirten Wassers bereitet, wurden stark abgekühlt und darauf in einem mit Eis amgebenen Gefässe gemischt. Es entstand ein blauweisser Niederschlag, der auf ein mit Eis umgebenes Filter gebracht und mit eiskaltem Wasser ausgewaschen wurde. Die Filtration geschah mittelst der Bunsen'schen Saugpumpe.

Wir werden nun die speciellen Versuchsdaten anführen und zunächst A. Filtrat und Waschwasser betrachten, da schon die Untersuchung derselben für die Frage über die Existenz der Salkowski'schen Verbindung entscheidend ist. Dann werden wir B. die Zusammensetzung der Niederschläge untersuchen, um Aus- 
kunft dariber zu erhalten, ob man berechtigt ist anzunehmen, dass dieselben eine chemisehe Verbindung von Tranbenzucker mit Kupferoxydhydrat enthalten.

\section{A. Untersuchung der Filtrate und Waschwasser.}

Vers. 1. Eine wässxige Lösung von $0.9 \mathrm{gr}$ Zucker wurde mit 6.235 gr Kupfervitriol und $50 \mathrm{ccm}$ normaler Kalilauge versetzt. Der gebildete Niederschlag war blauweiss, Filtrat farblos, änsserst schwach alkalisch, enthielt keine Spur von Kupfer, schwefelsaures Kali dagegen in reichlicher Menge und Zucker. Das Filtrat betrug $186 \mathrm{ccm}$, sein Zuckergehalt $0.050 \%$, es enthielt also $0.093 \mathrm{gr}$ Zucker oder mehr als $10 \%$ der angewendeten Menge. Der Niederschlag wurde nun in 3-4 Tagen mit eiskaltem Wasser ausgewaschen und der Zuckergehalt der durchgelaufenen Flüssigkeit bestimmt. Das erste Waschwasser, welches wir als zweites Filtrat bezeichnen wollen, betrug $209 \mathrm{ccm}^{1}$ ) und enthielt $0.0423 \mathrm{gr}$ Zucker. Während dieses Auswaschens war eine Aenderung der Farbe des Niederschlags von blau zu grün, besonders an der Oberfläche deutlich bemerkbar. Das dritte Filtrat, $202 \mathrm{ccm}$, enthielt 0.0293 gr Zucker, das vierte, $190 \mathrm{ccm}, 0.0235 \mathrm{gr}$. In diesem Filtrate liessen sich fortwährend noch Spuren ron Schwefelsäure nachweisen; das fünfte dagegen war frei davon; es betrug $217 \mathrm{~cm}$ und enthielt $0.0173 \mathrm{gr}$ Zucker. Es liess sich also noch Zucker auswaschen. Wir hielten jetzt jedoch mit dem Auswaschen ein, aber keineswegs aus dem Grunde, dass schon aller Zucker entfernt wäre, sondern weil der Niederschlag allmählich in so hohem Grade seine Farbe von blau zu grün veränderte (Reduction des Kupferoxydhydrats), dass wir eine $\mathrm{zu}$ weit gehende Decomposition desselben befürchteten.

Die Summe der in sämmtlichen Filtraten enthaltenen Zuckermengen ist $0.2054 \mathrm{gr}$ oder $22.8 \%$ der angewendeten Quantität. Wollte man aus diesem Ergebnisse das Molekularverhältniss zwischen der im Niederschlage zurückgebliebenen Zuckermenge und dem in demselben enthaltenen Kupferoxyd berechnen, würde man $1 \mathrm{Mol}$. Zucker auf $6.48 \mathrm{Mol}$. Kupferoxyd erhalten.

Vers. 2. $0.9 \mathrm{gr}$ Traubenzucker wurde in Wasser gelöst, mit einer Lösung von $6.235 \mathrm{gr}$ Kupfervitriol versetzt und $25.66 \mathrm{ccm}$ einer $7.8 \%$ igen Natronlösung zugefügt.

Das neutrale und farblose Filtrat war ganz kxpferfrei, enthielt aber Zucker. Filtrat 1, $222.5 \mathrm{ccm}$, enthielt davon $0.0648 \mathrm{gr}$; Filtrat 2 (das erste Waschwasser), $300 \mathrm{ccm}, 0.0378 \mathrm{gr}$; Filtrat $3,260 \mathrm{ccm}, 0.0227 \mathrm{gr}^{2}$ ); Filtrat 4, $220 \mathrm{ccm}, 0,0424 \mathrm{gr}$; Filtrat $5,400 \mathrm{ccm}, 0.0518 \mathrm{gr}$; Filtrat $6,320 \mathrm{ccm}, 0.0205 \mathrm{gr}$.

1) Wenn, wie in diesem Falle, die Zuckermenge im Filtrate sehr klein war, wurde die Flüssigkeit vor der Titrirung concentrirt.

2) Der Niederschlag auf dem Filter wurde mit einem Glasstabe umgerührt, bevor das Auswaschen fortgesetzt wurde. 
Dann wurde der Niederschlag in kaltem Wasser umgerührt und wieder auf das Filter gebracht; er enthielt fortwährend Zucker, der sich auswaschen liess, denn das 7. Filtrat enthielt $0.048 \mathrm{gr}$. Die Summe der ausgewaschenen Zuckermengen erreicht $0.288 \mathrm{gr} .32 \%$ der angewendeten Menge war also in Filtrat und Waschwasser übergegangen. Die im Niederschlage zurückgehaltenen 0.612 gr verhielten sich demnach zur gebrauchten Kupferoxydmenge wie $1 \mathrm{Mol}$ : $7.35 \mathrm{Mol}$.

Die folgende zwei Experimente, in welchen wir zuerst Alkali und dann Kupferlösung zusetzten, ergaben im Wesentlichen dieselben Resultate.

Vers. 3. 0.9 gr Traubenzucker, in Wasser gelöst, wurden in der Kälte mit $50 \mathrm{ccm}$ normaler Kalilauge und dann mit einer wässrigen Lösung von 6.235 gr Kupfervitriol versetzt. Der Niederschlag war blauweiss, das Filtrat farblos, äusserst schwach alkalisch, es enthielt keine Spur von Kupfer, sondern Zucker, indem eine kleine Probe die Fehling'sche Flüssigkeit deutlich reducirte. Filtrat 1, $117 \mathrm{ccm}$, enthielt $0.0869 \mathrm{gr}$ Zucker; Filtrat 2, $179 \mathrm{ccm}$, 0.0428 gr. Es waren jetzt 16 Stunden seit der Fällung verflossen; der Niederschlag war noch beinahe unverändert; er fing jedoch an, hie und da eine grünliche Färbung anzunehmen. Filtrat 3, $160 \mathrm{ccm}$, enthielt $0.038 \mathrm{gr}$ Zucker; Filtrat 4, $175 \mathrm{ccm}, 0.0259 \mathrm{gr}$; Filtrat 5, $195 \mathrm{ccm}, 0.0188 \mathrm{gr}$. Das Auswaschen wurde nun, um weiterer Decomposition za entgehen, eingestellt.

Die Gesammtmenge des ausgewaschenen Zuckers war in diesem Versuche 0.2124 gr oder 23.6 ${ }_{0}{ }_{0}$ der angewendeten Quantität. Das Molekularverhältniss zwischen Zucker und Kupferoxyd in dem ausgewaschenen Niederschlage liess sich demgemäss auf $1: 6.54$ berechnen.

Vers. 4. $0.9 \mathrm{gr}$ Traubenzucker wurden in Wasser gelöst und mit $25.66 \mathrm{ccm}$ einer $7.8 \%$ igen Natronlauge und einer Lösung von $6.235 \mathrm{gr}$ Kupfervitriol versetzt. Filtrat ganz farblos und neutral, völlig kupferfrei, gab aber starke Zuckerreaction. Filtrat 1, $225 \mathrm{ccm}$, enthielt $0.0778 \mathrm{gr}$ Zucker; Filtrat 2, $400 \mathrm{~cm}, 0.0433 \mathrm{gr}$; Filtrat 3, $260 \mathrm{ccm}, 0.0265 \mathrm{gr}$; Filtrat 4, $250 \mathrm{ccm}$, $0.024 \mathrm{gr} ;$ Filtrat 5, $380 \mathrm{ccm}, 0.0218 \mathrm{gr}$; Filtrat 6, $300 \mathrm{ccm}, 0.0156 \mathrm{gr}$. Der Niederschlag wurde nun in eiskaltem Wasser umgerührt, wodurch $0.022 \mathrm{gr}$ Zucker ausgezogen wurden. Die gesammte Zuckermenge in Filtrat und Waschwasser war also $0.231 \mathrm{gr} .=\mathbf{5 2} . \mathbf{7} \%$ der angewendeten Menge. Das Verhältniss zwischen Zucker und Kupferoxyd im Niederschlage nach dem Auswaschen würde also auf 1 Mol.: 6.73 Mol. zu berechnen sein.

Aus diesen Versuchen erhellt, dass 5 Mol. Kupferoxydhydrat nicht 1 Mol. Zucker zuriickzuhalten vermögen; es liess sich immer Zucker im Filtrate nachweisen, auch wenn man tagelang mit eiskaltem Wasser auswusch. Andererseits aber ist erwiesen, dass eine grössere Menge des Zuckers, die vorläufig auf etwa $3 / 4$ Mol. geschätzt werden kann, von den niedergeschlagenen 5 Mol. Kupferoxydhydrat hartnäckig zurückgehalten wird. Die aus den Versuchen berechneten Zahlen für das. 
Molekularverhältniss des Zuckers zum Kupferoxydhydrat im Niederschlage schwanken nämlich zwischen 1:6.48 und 1:7.35 ${ }^{1}$ ).

Vielleicht könnte man einwenden, dass die Kohlensäure der angewendeten Alkalilange einen Theil des Kupferoxyds (und zwar denjenigen, der als kohlensaures Salz ausgefällt werden müsste) daran verbindert hätte, eine Verbindung mit dem Zucker einzugehen, und dass aus diesem Grunde ein Theil des Zuckers im Filtrat erscheinen wïrde. Aber diese Menge müsste minimal gewesen sein, da die Flüssigkeiten nur Spuren von Kohlensäure enthielten. Um indessen diesen Einwurf ganz zu beseitigen, setzten wir in einigen besonderen Versuchen zu 1 Mol. Zucker 6 resp. 7 Mol. $\mathrm{CuSO}_{4}$ und 12 resp. $14 \mathrm{Mol}$. KOH; es war immer noch Zucker im Filtrate momentan nachweisbar. Diese Versuche entkräften selbstverständlich auch die Annahme, dass die Kohlensäure der Luft allmäblich auf die Verbindung von Kupferoxyd mit Zucker zersetzend eingewirkt habe, und dass es der so freigewordene Zucker sei, der sich auswaschen lasse.

Wie konnte nun aber ein so tiichtiger Forscher wie Herr Salkowski zu dem Resultate gelangen, dass der Niederschlag eine Verbindung ron 1 Mol. Zucker mit 5 Mol. Kupferoxydhydrat sei? Wir kömnen die Erklärang nur darin suchen, dass er seine Versuche zuerst mit diabetischem Harn angestellt hat; er beginnt nämlich seinen Aufsatz folgendermaassen ${ }^{2}$ ): „Wenn man bei Anstellung der Trommer' schen Probe mit diabetischem Harn im 'Zusatz des Kupfersulphats etwas unvorsichtig ist, so dass ein Niederschlag entsteht, der sich nicht wieder auflöst, so erhält man mitunter ein farbloses, schwach alkalisches, sowohl kupfer- wie zuckerfreies Filtrat. In anderen Fällen enthält dasselbe eine Spur Zucker, der grösste Theil jedoch bleibt im Niederschlag und wird von demselben auch bei noch so langem Auswaschen hartnäckig zurïckgehalten." Das so erhaltene Filtrat kann scheinbar zuckerfrei sein, weil man bei der gewöhnlichen Weise, in welcher die Trommer' sche Probe mit diabetischem Harn angestellt wird, oft nicht einmal $1 / 4-1 / 8 \%$ Zucker mit Sicherheit nachweisen kann.

1) $1: 6.48$ und $1: 6.54$ (Vers. 1 und 3) müssen übrigens als weniger genau betrachtet werden, da in diesen beiden Versuchen das Auswaschen unterbrochen werden musste, bevor es schon fertig war.

2) 1. c. S. 220 . 
Salkowski giebt weiter an, dass der Niederschlag, der auf 1 Mol. Zucker 5 Mol. Kupferoxydhydrat enthalten soll, sich in Natronlauge löst, dass diese Lösung beim Kochen redueirt wird, und glaubt sich nun im Stande, den Process und die quantitativen Verhältnisse bei der Trommer'schen Probe zu erklären: „Die Trommer'sche Reaction verläuft somit in zwei Phasen: in der Bildung der oben angegebenen Verbindung und in Auflösung derselben in der überschüssigen Kalilauge, welche bald zersetzend einwirkt" 1 ). Die angegebene Verbindung ist, wie wir eben gesehen haben, nicht nachgewiesen, wesshalb auch die von Salkowski gegebene Beschreibung des Verlaufs der Reaction, wie sie in den Lehrbïchern von Neubauer u. Vogel ${ }^{2}$ ) und Hoppe-Seyler ${ }^{3}$ ), aufgenommen ist, nicht ganz correct sein kann.

Durch die beschriebenen Versuche war also der Beweis geliefert, dass sich jene Verbindung Salkowski's unter den von ihm angegebenen Bedingungen nicht darstellen lässt, und insofern konnte man die Untersuchung als abgeschlossen betrachten. Da es aber darauf ankam, das Verhalten des Zuckers zu Kupferoxydhydrat überhaupt zu ermitteln, war es von Interesse, auch die Niederschläge näher zu untersuchen. Es war nämlich in denselben eine grössere Menge Zucker zurïckgehalten, und man konnte desshalb vermuthen, dass derselbe in chemischer Verbindung mit dem Kupferoxydhydrat stehe.

B. Untersuchung der Niedersehäge.

Bevor wir zur Beschreibung dieser Untersuchung übergehen, ist es nothwendig zu bemerken, dass die Niederschläge der niedrigen Temperatur ungeachtet sich zum Theil unter Abseheidung von etwas Kupferoxydul allmählich zersetzten.

Um zu ermitteln, ob die Niederschläge Zucker in chemischer Verbindung mit dem Kupferoxyd enthielten oder nicht, fanden wir es am richtigsten, ihre Zusammensetzung an verschiedenen Stellen zu untersuchen. Sollten nämlich in sämmtlichen Versuchen die Niederschläge iuberall die gleiche Zusammensetzung zeigen, wäre es wahrscheinlich, dass wir vorzugsweise mit einer chemischen Verbindung zu thun haben.

1) 1. c. S. $221-222$.

2) Anl. z. qual. u. quant. Anal. d. Harns. 7. Aufl. Wiesbaden 1876. S. 82.

3) Handb. d. physiol.- u. pathol.-chem. Anal. 4. Aufl. Berlin 1875. S. 122. 
Wir werden die Niederschläge in der den Versuchen entsprechenden Ordnung behandeln.

Vers. 1. Der Niederschlag wurde in zwei Parthieen getheilt; die eine blieb feucht, die andere wurde bei gewöhnlicher Temperatur in folgender Weise getrocknet. Sie wurde unter eine Glocke gebracht, durch welche mittelst der Bunsen'schen Pumpe ein Luftstrom gesaugt, der zuerst durch concentrirte Schwefelsäure, dann durch wasserfreie Phosphorsäure gestrichen war. Etwa mitgerissene Theilchen der letzteren wurden in einer mit Baumwolle gefülliten Röhre zurückgehalten.

Der feuchte Theil des Niederschlags wurde wieder in zwei Theile, deren jeder in Salzsäure gelöst und mit Schwefelwasserstoff gefällt wurde, getheilt. Die Kupferbestimmung geschah nach der Rose'schen Methode (Fresenius, Quantit. Anal. 6. Aufl. 1875. I. S. 334) und der Zucker wurde in dem von Schwefelwasserstoff befreiten Filtrate mit Fehling'scher Lösung bestimmt. In dem einen Theile war das Molekularverhältniss zwischen Zucker und Kupferoxyd 1: 9.3, im anderen ebenfalls 1:9.3.

Der trockne Niederschlag wurde ganz auf dieselbe Weise behandelt; in dem einen Theile wurde $1 \mathrm{Mol}$. Zucker auf 11.9 Mol. Kupferoxyd und im anderen 1:5.8 gefunden. Die letztere Zahl ist grösser als man voraus hatte vermuthen können; denn nach der Berechnung vom Filtrate sollte das Verhälťniss wie $\mathbf{1}: 6.48$ gewesen' sein $\left.{ }^{1}\right)$.

Vers. 2. In diesem Versuche musste man im Niederschlage das Verhältniss $1 \mathrm{Mol}$. Zucker a uf 7.35 Mol. Kupferoxyd erwarten; statt dessen wurde aber auf einem Orte $1: 16.15$ und auf einem anderen $1: 18.6$ gefunden.

Vers. 3. Auf einer Stelle wurde im Niederschlage 1 Mol. Zucker a uf 7.8 Mol. Kupferoxyd, auf einer zweiten 1:10.7\% und auf einer dritten 1: 15.08 gefunden. Man hätte 1: 6.54 erwarten müssen.

Vers. 4. Hier wurden 1:15.3 resp. 1: 10.3 gefunden statt (nach der Berechnung vom Filtrate) 1: 6.73.

Aus diesen Versuchen geht mit einer gewissen Wahrscheinlichkeit hervor, dass der vom Kupferoxydhydrat mitgerissene Zucker kaum in einem bestimmten Molecular-Verbältnisse zu m Kupferoxydhydrate stehen $\mathrm{kann}$, weil die Niederschläge im Ganzen eine wechselnde Zusammensetzung auf den verschiedenen Stellen hatten. Hieraus darf man aber jedoch keinen entschieidenden Schluss ziehen, weil eine grössere Menge des Zuckers zersetzt worden war. Diese Menge wurde in Versuch 1, in welchem sowohl die Kupfer- als die Zuckermenge im ganzen

1) Dieser Unterschied kann nur davon herrühren, dass dieser Theil des Niederschlags wenig zersetzt und der Zucker nur unvollständig ausgewaschen war. 
Niederschlag bestimmt wurde, festgestellt; $26.9 \%$ der angewendeten Zuckermenge war destruirt; cfr.:

Es waren angewendet . . . . . . . . . . 0.9 gr Zucker.

Es wurden wiedergefunden:

In Filtrat und Waschwasser . . $0.2054 \mathrm{gr}$

Im feachten Niederschlage . . 0.1940 "

Im getrockneten Niederschlage . 0.2598 "

Im Ganzen . . $0.6583 \mathrm{gr}$

Zersetzt waren also . . . . . . . . . $0.2417 \mathrm{gr}$

oder $26.9 \% 1$ ).

Ehe wir uns eine bestimmte Meinung über das Verhalten des Zuckers zum Kupferoxydhydrat bildeten, fanden wir es wünschenswerth, einen letzten Versuch anzustellen, bei welchem wir besonders bemuiht waren, die Zersetzung des Niederschlags möglichst zu verhindern. Das Resultat war aber anch in diesem Falle ganz dasselbe.

Vers. 5. Der mit Eis abgekühlten Zuckerlösung (0.225 gr Traubenzucker enthaltend) wurden unter Umrühren zuerst Natronlauge $(6.41 \mathrm{com}$ einer $7.8 \%$,igen $\mathrm{NaOH}$-lösung), dann die Kupfervitriollösung (1.55875 gr $\mathrm{CuSO}_{4}+$ $5 \mathrm{H}_{2} \mathrm{O}$ entsprechend) zugesetzt. Der dadurch gebildete Niederschlag war blauweiss; Filtrat ganz farblos, von neutraler Reaction, kupferfrei, gab aber deutliche Zuckerreaction und enthiclt schwefelsaures Natron. Der Niederschlag wurde auf dem Filter mit Eis umgeben und mit eiskaltem Wasser ausgewaschen, so dass seine Temperatur während des Versuchs niemals $7^{\circ}$ übertraf; im Allgemeinen war dieselbe nur $3-5^{0} \mathrm{C}$.

A. Filtrat.

1. Filtrat, $153 \mathrm{ccm}$, enthielt $0.0243 \mathrm{gr}$ Zucker. Das Auswaschen wurde fortgesetzt, bis das Waschwasser keine Reaction auf Zucker oder Schwefelsäure mehr zeigte. Es war dies nach der Anwendung von $940 \mathrm{ccm}$ Waschwasser der Fạll. Dasseibe wurde nun auf dem Wasserbade zu $185 \mathrm{~cm}$ concentrirt and mit der Fehling'schen Flüssigkeit titrit. Es enthielt $0.0318 \mathrm{gr}$

1) Die im Niederschlage gefundene Kupfermenge entsprach genau der angewendeten. Es wurden nämlich gefunden:

In feuchtem Niederschlage:
a) . . . . . . $0.2289 \mathrm{gr} \mathrm{Cu}$

b) . . . . . . $0.4098 \%$ "

Im getrocknetem :

a) . . . . . . $0.8720 \% "$

b) . . . . . . . $0.1026 \%$ "

$1.6133 \mathrm{gr}$ Cu statt $1.585 \mathrm{gr}$. 
Zucker. Im Ganzen liessen sich also aus dem Niederschlage $0.0561 \mathrm{gr}$ oder $24.93 \%$ der gebrauchten Zuckermenge auswaschen.

B. Der Niederschlag.

Dem auf einzelnen Stellen, besonders in der Mitte, grüngelben Niederschlag wurden drei Proben entnommen und in jeder derselben der Zucker und das Kupfer quantitativ bestimmt.

a) Die erste Probe wurde aus dem oberen Theil des Niederschlags genommen. Sie ergab $0.1419 \mathrm{gr} \mathrm{Cu}_{2} \mathrm{~S}=0.11331 \mathrm{gr} \mathrm{Cu}$. Filtrat enthielt 0.03136 gr Zucker. Das moleculare Verhältniss zwischen Zucker und Kupfer oxyd in dieser Probe war also $1: \mathbf{1 0 . 2 6}$.

b) Die $z$ weite Probe wurde aus der Mitte des Niederschlags genommen. Sie lieferte $0.1146 \mathrm{gr} \mathrm{Cu}_{2} \mathrm{~S}=0.0915 \mathrm{gr}^{\prime} \mathrm{Cu}$ und enthielt 0.02492 gr Zucker. Molekularverbältniss also $1 \mathrm{Zucker}$ a uf $10.42 \mathrm{Kupferoxyd}$.

c) Die dritte Probe war der untere Theil des Niederschlags. Sie enthielt $0.1024 \mathrm{gr} \mathrm{Cu}\left(0.1282 \mathrm{gr}^{\mathrm{Cu}} \mathrm{Cu}\right)$ und $0.0326 \mathrm{gr}$ Zucker, so dass das Molecularverhältniss $=1 \mathrm{Zucker}: 8.92 \mathrm{Kupferoxyd}$ gefunden wird.

d) Das Filter mit den daran haftenden Theilen des Niederschlags wurde mit verdünnter Salzsäure behandelt, und in der Lösung die Kupfermenge, die $0.08245 \mathrm{gr}\left(0.1033 \mathrm{gr} \mathrm{Cu}_{2} \mathrm{~S}\right.$ ) betrug, und der Zucker, der $=0.01763 \mathrm{gr}$ gefunden wurde, bestimmt. Wird das Molecularverhältniss zwischen Zucker and Kupferoxyd berechnet, findet sich 1: 13.28.

Im Ganzen wurden gefunden:

$$
\text { Kupfer. Zucker. }
$$

a) . . $0,11331 \mathrm{gr} .+0.03136 \mathrm{gr}$

b) . . . $0.09150 \%$. . . $0.02492 \%$

c) . . $0.10240 " . .0 .03260 "$

d) . . . $0.08245 \%$. . . $0.01763 "$

1. Filtrat. . . . . . . . . $0.02433 n$

2. $" . . .0 . . .0 .03179$,

0.38966 gr Kupfer und 0.16263 gr Zucker

statt $0.39625 \% " \quad 0.225 \%$ "

Während alles Kupfer wiedergefunden wurde, waren also $27.72 \%$ des Zuckers destruirt.

Das Ergebniss war also dasselbe wie in den früheren Ver" suchen.

Diese Thatsachen dïrten zur Entscheidung der Frage ausreichen, ob der Niederschlag nur als eine mechanische Mischung oder als eine chemische Verbindung zu betrachten sei.

Was für das letztere sprechen könnte, wäre einzig and allein die Farbenveränderung des Niederschlags von blau zu grün (und gelb), die von der Reduction des Kupferoxyds bedingt ist. Eine solche tritt nämlich nicht ein, wenn eine Traubenzuckerlösung 
mit Kupferoxydhydrat, selbst frisch gefälltem digerirt wird. Man darf aber hieraus keinen bindenden Schluss ziehen; die Verhältnisse sind in unseren Versuchen andere. Bringt man nämlich vorher gefälltes Kupferoxydhydrat in eine Zuckerlösung ein, kann die Berührung zwisehen beiden Körpern nicht eine so intime werden, als wenn der Zucker vom amorphen Oxydhydrat im Entstehungsmomente mitgerissen wird. Die Mischung der feinsten Theilchen, die in dem letzteren Falle erhalten wird, ist so innig, dass man sich das Eintreten einer chemischen Wechselwirkung zwischen dem Zucker und dem feuchten Kupferoxydhydrat als Base and zugleich sanerstoffabgebendes Mittel leicht vorstellen kann. Jedenfalls spricht jene Decomposition des Niederschlags mehr gegen als für die Auffassung desselben als chemische Verbindung.

Ein anderer Umstand schien uns aber sogleich zu Gunsten der Betrachtungsweise, dass der Niederschlag nur eine mechanische Mischung sei, fast entscheidend zu sein.

Wenn man nach mehrtägigem Auswaschen des Niederschlags, so dass das Waschwasser nur noch minimale Mengen Zucker enthielt, denselben in kaltem Wasser suspendirte (vergl. Vers. 2 u. 4), so nahm die Zuckermenge im Filtrate zu. Es kann dies nur darauf beruhen, dass der Zucker mechanisch beigemiseht ist, und zeigt, dass er denjenigen Stoffen angehört, die relativ leicht von Niederschlägen festgehalten werden. Dieses findet auch in anderweitigen Erfahrungen eine Stütze. Der in Lösung befindliche Zucker wird von Thierkohle zuriickgehalten, aus welcher er sich indessen dureh längeres Auswaschen entfernen lässt, aber, wie es scheint, auch dann nur unvollständig; und beim Zusatz von Bleiessig zum zuckerhaltigen Harn wird, wie Brỉcke gezeigt hat, ein Theil des Zuckers vom Niederschlage mitgerissen. Andererseits weiss man, dass Kupferoxydhydrat beim Ausfällen auch andere gelöste Stoffe, nämlich Alkalihydrate, zurückhalten kann, die sich bekanntlich dann nicht vollständig durch Auswaschen entfernen lassen. Als Beispiel dafür, wie hartnäckig Niederschläge mechanisch mitgerissene, an sich lösliche Stoffe festhalten können, wollen wir nur daran erinnern, wie schwierig der schwefelsaure Baryt sich von Alkalisalzen befreien lässt.

Ein anderer und völlig entscheidender Beweis dafür, dass der Niederschlag wirklich nur eine mechanische 
Mischung ist, liegt darin, dass wenn man 1 Mol. Zucker 10, ja sogar $30 \mathrm{Mol}$. Kupfervitriol und 20 resp. $60 \mathrm{Mol}$. Kalihydrat zusetzt, das Filtrat noch relativ grosse Zuckermengen enthält.

Das letzte Resultat war uns übrigens unerwartet. Obgleich unsere Untersuchung zu einem negativen Resultate in Bezug auf die Frage von der Existenz einer chemischen Verbindung zwischen Zucker und Kupferoxydhydrat im Niederschlage fithrte, vermutheten wir doch, dass bei der Anwendung grösserer Quantitäten Kupfersulphat und Alkali kleine Zuckermengen vollständig vom Niederschlage zurïickgehalten werden dürften. Wäre dies der Fall, könnte es vielleicht gelingen, auf diesem Wege klein e Zuckermengen, z. B. in thierischen Flüssigkeiten, nachzuweisen. Man hätte dann im Wesentlichen nur nöthig, den Niederschlag abzufittriren, in einer Säure zu lösen und nun versehiedene Zuckerproben anzustellen. Die geringe Haltbarkeit des Niederschlags wirde, wenn man die Operationen schnell und in der Kälte ansführte, gewiss keine uniberwindliche Schwierigkeit bieten; allein die Thatsache, dass selbst ein sehr grosser Ueberschuss an Kupferoxydhydrat nicht allen Zucker bei der Ausfällung mitzureissen vermag, zeigt in genügender Weise die Unanwendbarkeit der Methode zu diesem Zweeke.

Schluss und Resumé. (In Wasser gelöster) Traubenzucker, mit wässrigen Lösungen von Kupfervitriol und Alkali, den letzteren in Aequivalentverhältnisse versetzt, wird in dem gebildeten Niederschlage mitgerissen und kann zum Theil aus demselben ausgewaschen werden. Der Niederschlag enthält keine chemische Verbindung von Kupferoxydhydrat mit Zucker, zersetzt sich aber doch leicht in feuchtem Zustande.

Der Traubenzueker wird aber nicht vollständig zurückgehalten, selbst wen man einen grossen Ueberschuss an Kupfervitriol und Alkali anwendet.

\& 2. Ueber die von Hoppe-Seyler und Fileti angegebenen Verbindungen von Traubenzucker mit Kupferoxyd.

Im Folgenden werden wir unterstuchen, ob irgend eine Verbindung zwischen Zucker und Kupferoxyd wirklich existirt. 
Hoppe-Seyler sagt ${ }^{1}$ ): „Eine wässrige Lösung von Traubenzucker löst reichlich Aetzkalk auf, ebenso auch Kupferoxydhydrat. Die dunkelblane Flüssigkeit, die man durch Aufösen von Kupferoxydhydrat in Traubenzuckerlösung erhält, ist jedoch sehr zersetzlich, schon nach kurzem Stehen scheidet sich ein gelbes oder rothes Pulver, Kupferoxydul, aus, während die Lösung sich entfärbt; hierbei wird der Zucker oxydirt, indem Ameisensäure, Oxymalonsäure, vielleicht auch Essigsäure und ein dem Dextrin ähnlicher Körper, entstehen," und dasselbe wiederholt er in der 4. Auflage, 1875, S. 122. Um dieses zu controliren, haben wir folgende Versuche angestellt:

1) $75 \mathrm{ccm}$ einer Traubenzuckerlösung, die $0.9 \mathrm{gr}$ Zucker enthielt, wurden mit $25 \mathrm{ccm}$ Wasser und ca. $2.5 \mathrm{gr}$ frisch gefällten Kupferoxydhydrat, nach Löwe's Methode durch Fällen einer Lösung von schwefelsaurem Kupferoxyd-Ammonialk mit Alkalilauge ${ }^{2}$ ) dargestellt, versetzt. Die Flüssigkeit wurde 24 Stunden lang, mit Eis ungeben, unter stetigem Umrühren auf einer kalten Stelle stehen gelassen; als nach Verlauf dieser Zeit keine Veränderung wahrzunehmen war, wurde die Flüssigkeit in ein Zimmer von etwa $5^{\circ} \mathrm{C}$. gebracht und nach 24 Stunden filtrixt. Das Filtrat war ganz farblos und enthielt keine Spuren von Kupfer. Das unveränderte Kupferoxydhydrat, das keine Andeutung zur Reduction zeigte, wurde mit etwas Wasser ausgewaschen, welches mit dem Filtrat vereinigt wurde. Im Filtrat und Waschwasser fanden sich im Ganzen 0.8275 gr Zucker. Der Niederschlag wurde dann in Wasser suspendirt, in Salzsäure gelöst und mit Schwefelwasserstoff gefällt. Das gebildete Schwefelkupfer wurde auf ein Filter gebracht, das Filtrat von Schwefelwasserstoff befreit und concentrirt. Es gab mit der Fehling'schen Flüssigkeit deutliche Reaction auf Zucker, enthielt aber davon so wenig, dass eine quantitative Bestimmung nicht gelang, obgleich nur $2 \mathrm{ccm}$ der Titrirflüssigkeit angewendet wurden.

2) Zu $48.1 \mathrm{ccm}$ Traubenzuckerlösung, die 0.9 gr enthielten, wurden 20 ccm Wasser und $2.91 \mathrm{gr}$ lufttrocknes Kupferoxydhydrat gesetzt. Nachdem die Flüssigkeit 2 Tage lang bei $30^{\circ} \mathrm{C}$. gestanden hatte, ohne dass Spuren von Kupfer sich lösten, wurde die Temperatur bis $40^{\prime}$ C. erhöht; das Resultat blieb aber dasselbe. Das Kupferoxydhydrat wurde abfiltrirt und ausgewaschen. Filtrat und Waschwasser enthielten im Ganzen 0.822 gr Zucker. Der Rüickstand auf dem Filter wurde in der bei dem vorigen Versuche angegebenen Weise behandelt; er enthielt Zucker, aber weniger als $0.025 \mathrm{gr}$.

3) Der dritte Versuch war eine Wiederholung des zweiten, nur mit dem

1) Handb. d. physiol.- u. pathol.-chem. Anal. 3. Auf. 1870. S. 108.

2) Vgl. Gmelin's Handb. d. anorg. Chem. 6. Aufl. Bd. 3. 1875. S. 599 u. Dingler's Polyt. Journ. 1858. Bd. 149. S. 270. 
Unterschiede, dass statt des lufttrocknen eine entsprechende Menge feuchtes, frisch gefälltes Kupferoxydhydrat angewendet wurde. Filtrat und das damit vereinigte Waschwasser enthielten 0.8 gr Zucker; das Kupferoxydhydrat hatte nur Spuren davon aufgenommen.

Diese Versuche zeigen mit Bestimmtheit, dass der Traubenzucker Kupferoxydhydrat nicht lost.

In den „Berichten der deutschen chemischen Gesellschaft zu Berlin" Bd. 8, 1875, S. 441, wird in einer Correspondenz aus Turin mitgetheilt, dass Fileti zwei sogenannte Kupferglycosate dargestellt hat, von welchen das eine die Formel $\mathrm{C}_{6} \mathrm{H}_{6} \mathrm{Cu}_{3} \mathrm{O}_{6}+$ $2 \mathrm{H}_{2} \mathrm{O}$ entsprechende Kapfermenge und das andere $64.48 \% \mathrm{Cu}$ enthaten soll, woraus die Formel $\left(\mathrm{C}_{6} \mathrm{H}_{7} \mathrm{O}_{6}\right)_{2}\left(\mathrm{Cu}_{2}\right)_{5}$ abgeleitet wird. Zwar entstehen unter den von Fileti angegebenen Umständen Kupfergly cosate, diese sind aber nicht Verbindungen von Zncker mit Kupferoxydallein; sie enthalten auch Kali. Sie sind also Doppelverbmdungen, und mit diesen werden wir uns in einer anderen Arbeit beschäftigen, worin wir das Verhalten des Zuekers zu Kupteroxyd in alkaliseber Lösung besprechen werden.

\title{
Fortgesetzte Untersuchungen über die Secretion der Niere.
}

Von

\section{Dr. Mority Nussbaum.}

\author{
Hierzu Tafel XII.
}

In Folgendem beabsichtige ich, die Resultate fortgesetzter Untersuehungen über die Secretion der Niere zu geben und die Bestimmung des Ortes der Ausscheidung der wichtigsten pathologischen Harnbestandtheile durebzufïhren. Zum besseren Verständniss der mitzutheilenden Versuche sei es erlaubt, zuror die 